\title{
Plagiarism Detection System for Armenian Language
}

\author{
Gevorg Margarov \\ National Polytechnic University of \\ Armenia \\ Yerevan, Armenia \\ gmargarov@gmail.com
}

\author{
Gohar Tomeyan \\ National Polytechnic University of \\ Armenia \\ Yerevan, Armenia \\ goharikyan93@gmail.com
}

\author{
Maria João Varanda Pereira \\ Polytechnic Institute of Bragança \\ Bragança, Portugal \\ mjoao@ipb.pt
}

\begin{abstract}
In the academic context, it is very important to evaluate the uniqueness of reports, scientific papers and other documents that are everyday disseminated on the web. There are already several tools with this purpose but not for Armenian texts. In this paper, a system to analyze the similarity of Armenian documents is presented. The idea is to collect a set of documents of the same domain in order to identify keywords. Then, based on that information, the system receives two documents and compares them calculating the probability of plagiarism. For that, an approach based on several levels of analysis is implemented and some of those steps allow the user interaction choosing options or adding more information.

Keywords-Natural language processing, plagiarism detection, sinonymizer, document uniqueness, document similarity.
\end{abstract}

\section{INTRODUCTION}

Internet is getting more widespread in our life and in our activities. However, visiting different Web sites, we see that all the found articles or other materials are very similar. Besides, there are many thesis, term papers, research and other scientific works on the Internet. If formerly it was necessary for the students to take advantage of the published books and literature, now it is enough to write the name of the subject in the search engines and we can find thousands of items. The most common objects of plagiarism are texts, separate expressions, thoughts, inventions, facts described in novels. Scientific spheres include a large amount of ready works, course works and articles, in which we can make several changes and achieve results. That kind of change is considered plagiarism. In order to avoid these situations a plagiarism detection system is needed.

At first will define what plagiarism means. There are many definitions of plagiarism. The scientific and educational sphere plagiarism is the form of deception, which means to appropriate other ideas, passages from another work or author. This is a forgery generally in violation of copyright laws. Plagiarism is a steal and pass off the ideas of another as one's own, using another's manufacture without lending the source, present as a new and original an idea taking from an existing source [1]. In the legal point, plagiarism is a direct privatization of the text. Legally the plagiarism is text digestion, while the digestion of subjects and ideas can't be considered as plagiarism. The only thing, which is not allowed, is the whole copy of the text. But often the whole text is translated and presented as an original. Thus, the plagiarism, which is done by translation is widespread.

Usually in order to conceal the plagiarism people carry out several steps, for example text morphological change, lexical change, reduction of the text up to some words, sentences, pictures or formulas, text syntactical changes, movement of the sentences, punctuation marks change, spaces are replaced with transparent letters, and also create and use synonyms.

With the ever-increasing availability and accessibility of the Internet, students are able to access a multitude of resources in support of their studies. However, this has also led to an increase in their ability to cheat through plagiarizing text and claiming it as their own. So, one of the most important part of this work is to define plagiarism levels and what must be checked in each level. Then, construct a tool implementing that plagiarism levels detection for document written in Armenian language.

There are already several tools with this purpose but not for Armenian text. Existing plagiarism detecting multilingual systems are not intended for Armenian language.

\section{RELATED WORK}

There are many automatic systems to detect plagiarism; such systems are Antiplagiat.ru, eTXT, PlagScan, CheckforPlagiarism.net [2], Turnitin, etc. Here we describe as comparison analysis of some textual softwares.

The most famous online system is Antiplagiat [3]. The system searches from its own database. Therefore, the system has several disadvantages. At first, it isn't able to search on the Internet and there is a limit up to 3000-5000 words. The system Antiplagiat doesn't detect text morfological changes. If spaces are replaced with transparent letters, they will be visible to computer. The system Antiplagiat is able to detect, reduced, and replaced words, sentences and paragraphs. The replacement of English letters with Russian is also detected. The change of punctuation marks has no influence on work of the system.

eTXT-Antiplagiat [4] gives the opportunity to search similar documents on the Internet. Matching parts of the text are indicated with the respective colors. It can easily detect 
non-unique texts. To avoid to be detected by the system we need to make changes in the text using synonyms, for instance.

PlagScan is a plagiarism detection software (available online and on-premises), used by academic institutions and businesses. PlagScan [2] servers teachers and professors to identify plagiarism and educate students on the appropriate usage of sources in academic works as well as protecting copyrights of texts. The main disadvantages of PlagScan are: it doesn't support synonym recognition, sentence structure checking and plagiarism detection over translated texts isn't supported.

Mainly all the systems use the algorithm of shingle, which provides the highest correctness in detecting the copies.

In this Fig. 1, the main characteristics of the tools are presented, allowing us to compare the facilities provided by each one. For example, there are tools that don't detect the use of synonym and only one tool can found the plagiarism with translation only from English texts, that is the Turnitin [5].

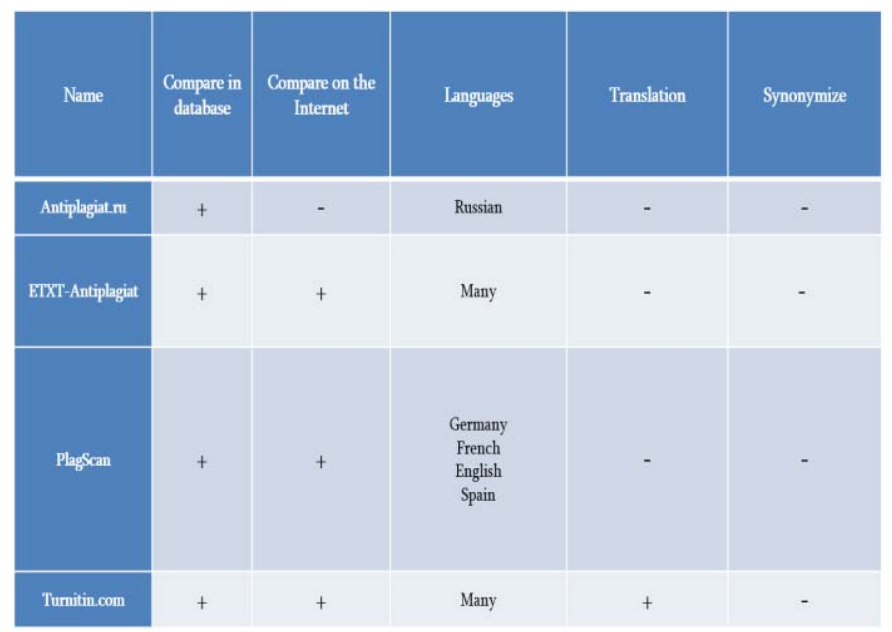

Fig. 1. Comparative analysis

To avoid such situations, it was decided to develop a system that will automate the uniqueness analysis of the work done by students in the learning process and will allow teachers to detect quickly the existence of plagiarism. There is not this kind of systems for Armenian language, this one will be used in lot of universities and will be useful for lecturers.

The main features of this work are:

- Checking in the database: The program will be check the students papers in the system database, where each year can be uploaded the research works done by students.

- Checking on the Internet: The system doesn't give opportunity for searching on the web sources, but teachers can upload web documents to prevent the plagiarism based on the Internet.

- Checking the use of synonyms and sentence structure changes: The system will allow the following steps: normalization alphabet, keyword detection, stop word removal, stemmer, which will be use to search the correct forms of words.

- Multiple Document Comparison: Our system will compare one document with more documents and will show the percentage of plagiarism possibility considering the keywords of the domain.

- Supported Languages: Armenian.

- Plagiarism with translation: The program will detect Russian and English text translations, and will compare with Armenian sources. The translation is based on the Google translator.

\section{PLAGIARISM CHECKER SYSTEM: OUR PROPOSAL}

To achieve the assigned goal it is necessary to solve the following tasks:

- review the existing algorithms for detecting plagiarism in the texts,

- review existing methods to conceal the fact of plagiarism, as well as methods of dealing with them,

- develop a method of searching plagiarism in Armenian texts that is resistant to possible text modifications,

- create a software tool based on the developed method, which provides plagiarism detection with the possibility of visualizing the borrowed pieces of text in the scanned document and in the source document. At the end, a percentage will be calculated in order to identify the document similarity level.

Below we will describe the main steps of our tools:

Natural Language Processing (NLP) techniques [6], are used to detect the possibility of plagiarism in Armenian texts. The main idea is to analyze the similarity between two documents using those techniques of natural language processing [7].

The first step will be to compare the texts word by word but this work must go further. Everyone knows that the people that use the texts of other people change it a little bit to dissimulate the plagiarism.

Natural Language Processing includes semantic and syntactic changes, stop word removal, stemming, lemmatization, punctuation removal and etc., as part of the preprocessing stage. If the text has semantic and syntactic changes, the plagiarism detection systems do not work well. In order to detect such changes, linguistic techniques must be considered. It's important to detect intelligent plagiarism, when ideas are presented in different words, replacement with synonyms, translation, etc. Translation plagiarism is also very common, because students can also translate the text from one language to another without pointing the original source. For 
example we haven't many materials about Information Systems in Armenian language and students carry out translation from English or Russian texts including automatic translation (for example Google and another translators) and manual translation (which can be done by students who knows some languages).

So, one of the most important part of this work is to define plagiarism levels and what must be checked in each level. Then, construct a tool implementing those plagiarism levels detection.

Possible modifications of the text plagiarism depends on the language used, and during the analysis of the text, we should take into account the specifics of the given language. Each language has different rules for sentence structure and different opportunities for synonym replacement.

Detecting plagiarism should be made by possible modifications when detecting, and the system must be able to allocate specific pieces of borrowed text, as well as the corresponding fragments of the source text. In order to process an algorithm, it is important to determine two aspects:

- standards of determining the similarity of texts (form and content),

- determining the level of similarity and its threshold value (when the text isn't a copy)

Technical uniqueness of text is a threshold value, which is usually measuring by percentage. The text that has an 100 percent technical uniqueness, is not unique yet (de facto it can be unique also from about 0). For example, write off the thought of another person, and that is not unique, measuring by other words. However, there are some exceptions too, factual unique texts can be a technical unique for 50 percent. For example, the author's work is unique, when includes included exceptional materials that are written from 0 . A work is not unique when it includes citations, expressions, technical terminus and etc.

The main steps of this work is to find sentences exactly the same, normalization alphabet, keywords detection, stop-word removal, stemming, synonym recognition and find plagiarism with translation. The steps of the plagiarism detection tools you can see in Fig. 2.

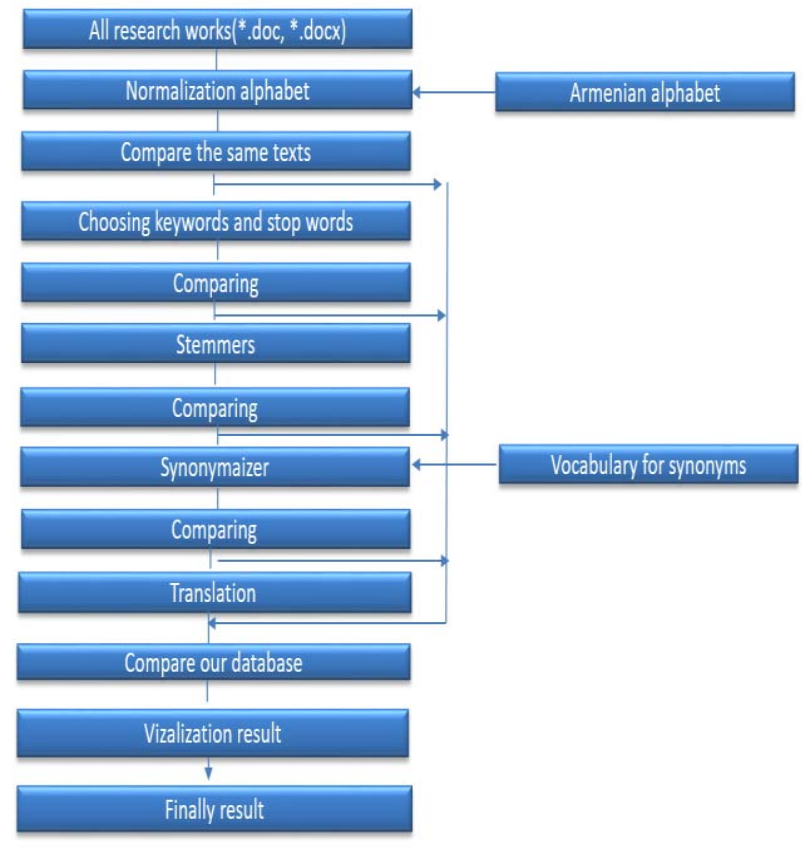

Fig. 2. Steps of plagiarism detection.

First step is to normalize alphabet and the second step is to find exactly the same texts. Then, removing the stop words and using a set of predefined keywords of the text domain the document should be compared again. Stop-words is a very frequent words but without any particular meaning. The usual way of determining what counts as a stop-word is just to use a dictionary that lists them [8]. And in our program we used the stemming system to delete endings in Armenian language. The motivation for using synonymy recognition comes from considering human behavior, whereby people may seek to hide plagiarism by replacing words with appropriate synonyms. The system also contains synonyms and steamers for the Armenian language.

The most significant principles are lexical analysis, as well as linguistic methods. To detect lexical changes we used steamers, which are based on Porters algorithm considering the features of Armenian language. The algorithm gives an opportunity to delete verb endings, noun ending and other types of etc. In our program is used the idea of keyword, which gives an opportunity to organize searching in our database very quickly. Keywords have special meaning and they are chosen and formed according to each subject. To find synonyms we use Armenian vocabulary. Now the Shingles method is also used which will give an opportunity in the future to carry out search on the Internet. At the end, in our program, we used the Google translator for finding plagiarism with translation.

\section{THE CREATED SYSTEM IN DETAIL}

We will represent the main steps to find exactly the same sentences, choosing keywords, stop word removal, synonyms recognition and translation for finding the possibility of plagiarism. The program only compares *.doc, *.docx 
documents in our database. Database will be expanded by teachers uploading and checking the student documents, as well as translated documents. The system allows to carry out searching based on previous years works. The steps of the plagiarism detection tools we can see in Fig. 2.

Often students can replace the letters with another letters, for example, some systems are not able to detect if there is Russian "a" letter instead of English "a" letter. We have some letters which are similar to another letters, for example Armenian "h" it seems like English "h". This program is able to find other letters and point out in another color.

In our program the alphabet is checked at first whether is it written in Armenian or not.

Checks are carried out through ASCII codes. If it is not Armenian, letters it will be pointed out in red color. The program includes the Armenian letters, and letters are comparing through the ASCII code. When the program point out letters, which are written in another language by red color, the teacher will see the result, will be able to replace the letters into Armenian but manually. After this taken steps, teacher can compare them. If teacher does not replace them, sometimes the system will not be able to recognize and will consider as another word. That is a main meaning of normalization alphabet.

First important part of plagiarism detection is to find exactly the same text. The program can find exactly the same text and show the percentage, whether there are matching parts. The comparison is realized word by word. At first for comparing, we need to delete all characters except the ".", which shows that the sentences are completed. This algorithm is used to split the source text into sentences. Separation is carried out by punctuation marks such as a point, exclamation mark, question mark then the text is compared sentence by sentence and if there is a match it will indicate plagiarism existence otherwise continues to perform the next action. The program can compare two or more files. If we want to compare many documents, we will need only to choose the subject, after that keyword are extracted and we can see how the possibility of plagiarism is. We can compare two or more documents.

After identifying the most important elements of papers, we have already keywords for each subject, which are kept in Microsoft Word and saved by special name, for example name of the subject. The program gives the solution for the teachers to upload a new file, which may contain his own keywords and synonyms of keywords or teachers can edit already existing keyword files. The program working like this, if we want to generate keyword for any subject, first we need to put password, and after choosing the name of the subject, or upload file. Depend on the fact that who will enter the password, opportunity of the user will be different. As administrator, the user can add a new keyword with the help of corresponding window, but a teacher doesn't have that kind of solution. After that we need to choose file, which we want to compare. The program will generate the new folder, and put there only those files that we have in our database and which have the same keywords. When we will compare we can see result presented by percentage. We do not need to compare all files, we only have to compare the text, which have the same keywords. Each subject has separately keywords that are kept in separate documents.

The program now includes 100 stop words for Armenian language and we can delete stop words, see frequency and compare. Stop words are saved in our database, and in the future, it will be extended.

One of such important and necessary things on computer linguistics is the operation of the using steamers. Stemming are usually used in Information Retrieval systems. Best way for determining steamer it is just using the dictionary. The project Snowball contains the old version Armenian suffix and prefix, but Armenian language has endings too, when in Armenian language we delete suffix or prefix, the words will change it's meanings. But for English language endings and suffix have same meanings. For example, if words finished in -ed, just in English we can delete "-ed" suffix and words will not change the meaning. In our program, teachers can see all endings. If teacher wants to compare two files and to know possibility of plagiarism, after he/she can delete all endings, needs only to choose the second "text endings" and compare. All endings will be deleted; therefore the program already gives the percent without endings.

The most important thing for NLP is identification of synonyms. The main concept is to use synonyms but to keep the meaning of the text. After using stemming we can replace with synonyms. The program has an option which points out words in red color and replace with synonyms. Teacher has the opportunity to point out words in red color, choose the meaning, which corresponds to the context and save changes. After choosing the word in the right side appears panel, where the user can see the meaning of synonyms and after choose corresponding word, a comparison will be done and a result by percentage will be calculated.

Teachers can add and see the synonyms, which are existing in our database. The teacher can only delete synonyms, which he/she wrote. At first teacher need to write synonym, explanation and choose the add button, after we can see all the explanation, if its correct teacher can write in database.

As already have been told, plagiarism can be done translating the text from one language to other without referencing to the original source. Translated plagiarism can include two type of translation: automatic and manual translation.

Plagiarism with translation is very difficult to detect. There are many kinds of problems: first is to translate words that have many meanings; the translator translates all words automatically, and the system has to find which one is correct word. A word by word translation is not a good idea. Translation for Armenian language is not working effectively, it's enough only for understanding but not for detection plagiarism, but Google gives an huge opportunity to make changes and optimize the texts returned by Google translator. We don't have much information in Armenian language on the Internet, and students often translate the documents from Russian and English texts, and present as own idea. Usually students use the already existing translators, especially Google 
translate. For that reason we include Google translate in our program, because the translator allows the translation of the documents. And translation will work if the user has connection to the Internet.

If we want to translate the document we need to choose the document, when program finished translation, teachers must copy and paste the text on the Microsoft Word, and after which upload that file to our database. And then the teacher can follow the same steps to detect plagiarism: choose keywords and compare with many documents or compare only two documents using stop word removal and synonym recognition.

\section{SYSTEM IMPLEMENTATION}

In order to implement the system a local database is used. Search for detecting should be carried out in the local database of documents. The implementation was done in the language $\mathrm{C}$ sharp, Windows Form Application for creating Desktop Application and Asp.net MVC for making Web Application. We used MSSQL (to work with the database) and Google translate for detecting plagiarism on the Internet with translation.

\section{TESTING}

We performed already some tests with real users and some conclusions were taken. We tested the system functionalities and also linguistic failures. The main disadvantage is retiled to hard interface of desktop application, which is very difficult to use without user guide. Another disadvantage is the system has very few synonyms, which will be added in near future or we will use the synonymizer for Armenian texts.

More tests will be carried out in order to measure the effectiveness of the system.

\section{CONCLUSION}

This paper described the proposed plagiarism detection system for Armenian documents. The system compares two and more documents and allows the following steps: normalization alphabet, keyword detection, stop word removal, steaming, and it is able to detect the replacement by synonyms and find plagiarism with translation. Our plagiarism detection system compares the texts in directory, which is extended owing to teacher's uploaded files. A Web application were also created, which will be extended and available not only for teachers but for all the users in the future.

\section{ACKNOWLEDGEMENT}

We are so grateful to Erasmus + ICM project for supporting the research collaboration between IPB and NPUA.

\section{REFERENCES}

[1] Amalia, "Performance Evaluation of Free Anti-plagiarism Software," Proceedings of The 3rd Annual International Conference Syiah Kuala University (AIC Unsyiah), October 2-4, 2013, pp. 30-36.

[2] A. M. El Tahir Al and Hussam M. Dahwa Abdulla, "Overview and comparison of plagiarism detection tools," In DATESO 2011, pp. 161-172.
[3] P. I. Mozgolyova, K. V. Gulayeva, and O. M. Zamyatina, "Information technologies for the assessment of competencies and organization of project activities at the training of technical Specialists", Informatization of Education and Science, , pp. 3046, 2013, (in russian).

[4] E. S. Cherkin. "Systems of automated verification for illegal matching," Bulletin of Tambov University. Series: The Humanities Scince, no. 12(128), pp. 164-174, 2013, (in russian).

[5] D. Weber-Wulff1, C. Möller1, J. Touras, and E. Zincke, "Plagiarism detection software test 2013", Abgerufen am, 2014, p. 12.

[6] S. Alzahrani, N. Salim, and A. Abraham. "Understanding plagiarism linguistic patterns, textual features, and detection methods", IEEE Transactions on Systems, Man, and Cybernetics, Part C (Applications and Reviews), vol. 42, Is. 2, pp. 133-149, 2011.

[7] M. El Bachir Menai, "Detection of plagiarism in arabic documents," International Journal of Information Technology and Computer Science(IJITCS), vol.4, pp. 80-89, Sep. 2012.

[8] Z. Ceska and C. Fox, "The Influence of Text Pre-processing on Plagiarism Detection", International Conference on Recent Advances in Natural Language Processing, pp. 55-59, 2011. 